\title{
Improved accuracy for the detection of sleep apnea-related surges in blood pressure
}

\author{
Ryujiro Sasanabe and Toshiaki Shiomi \\ Hypertension Research (2011) 34, 909; doi:10.1038/hr.2011.87; published online 30 June 2011
}

$\mathrm{T}$ he severity of obstructive sleep apnea syndrome (OSAS) increases in obese individuals along with the prevalence of complications, such as hypertension, type- 2 diabetes, metabolic syndrome and chronic kidney disease, ${ }^{1,2}$ and these health-related issues lead to an increased risk of cardiovascular and cerebrovascular disease. ${ }^{3}$

Regarding the effects of OSAS on the cardiovascular system, patients with severe OSAS who continue treatment with continuous positive airway pressure (CPAP) have an improved prognosis, whereas patients who cannot continue CPAP have a clearly worse prognosis. ${ }^{4}$ In addition, another study has shown a high incidence of nocturnal deaths in OSAS patients. ${ }^{5}$

To prevent cardiovascular and cerebrovascular disease, risk factors such as metabolic syndrome and chronic kidney disease must be diagnosed early. Blood pressure must be controlled, which involves not only lowering the blood pressure but also minimizing wide fluctuations in blood pressure.,

Among middle-aged patients with OSAS, many patients without hypertension during the daytime may exhibit large variations in blood pressure and pulse rates only when breathing resumes by arousal reaction after nocturnal apnea. However, recording these surges in blood pressure at night is difficult with conventional ambulatory blood pressure monitoring (ABPM), which measures blood pressure at fixed intervals. Accordingly, Shirasaki et al. ${ }^{8}$ developed an ABPM method that detects arterial oxygen desaturation due to apnea and from that point measures

Dr R Sasanabe and Professor T Shiomi are at the Department of Sleep Medicine, Aichi Medical University School of Medicine, Aichi, Japan.

E-mail: sasanabe@aichi-med-u.ac.jp blood pressure. However, the measurement algorithm used by Shirasaki et al. ${ }^{8}$ was weakly correlated with apnea severity, including the duration and severity of apnea itself and the severity of oxygen desaturation.

To overcome this deficiency, in another paper, Shirasaki et al. ${ }^{9}$ proposed to further measure the blood pressure when the degree of oxygen desaturation became even more severe, without setting times when the blood pressure was not measured. This approach offered the advantage of blood pressure measurements corresponding to times when there was severe oxygen desaturation-namely, when there might be a reactive increase in blood pressure. However, by removing fixed times when the blood pressure was not measured, frequently repeated blood pressure measurements could interrupt sleep maintenance. To reduce the frequency of blood pressure measurements, the threshold for oxygen desaturation must be set to large values $\geqslant 10 \%$.

The severity of OSAS is evaluated by the apnea-hypopnea index. According to the American Academy of Sleep Medicine Manual for Scoring of Sleep and Associated Events, ${ }^{10}$ hypopnea is defined as a respiratory event associated with $\geqslant 4 \%$ oxygen desaturation. Currently, the degree of oxygen desaturation required to initiate the blood pressure measurement is $10 \%$. The difference between 4 and $10 \%$ is large, and thus a considerable number of hypopnea and apnea events are being missed. It is possible that only severe events are being detected, which is a drawback to this approach. Surges in blood pressure in patients with slight arterial oxygen desaturation, such as elderly women with mild heart failure, are probably not being detected. Obese middle-aged patients with OSAS primarily exhibit snoring and have a short history of hypertension.
Therefore, a revision of this algorithm may enable the more accurate measurement of nocturnal changes in blood pressure compared with conventional ABPM.

\section{CONFLICT OF INTEREST}

The authors declare no conflict of interest.

1 Sasanabe R, Banno K, Otake K, Hasegawa R, Usui K, Morita M, Shiomi T. Metabolic syndrome in Japanese patients with obstructive sleep apnea syndrome. Hypertens Res 2006; 29: 315-322.

2 Iseki K, Koharura K, Sakima A, Iseki C, Kinjo K, Ikemiya $Y$, Takishita $S$. Changes in the demographics and prevalence of chronic kidney disease in Okinawa, Japan (1993-2003). Hypertens Res 2007; 30: 55-62.

3 Shamsuzzaman AS, Gerh BJ, Somers VK. Obstructive sleep apnea: implications for cardiac and vascular disease. JAMA 2003; 290: 1906-1914.

4 Marin JM, Carrizo SJ, Vicente E, Agusti AG. Long-term cardiovascular outcomes in men with obstructive sleep apnoea-hypopnoea with or without treatment with continuous positive airway pressure: an observational study. Lancet 2005; 365: 1046-1053.

5 Gami AS, Howard DE, Olson EJ, Somers VK. Day-night pattern of sudden death in obstructive sleep apnea. N Engl J Med 2005; 352: 1206-1214.

6 Kario K, Pickering TG, Umeda Y, Hoshide S, Hoshide Y, Morinari M, Murata M, Kuroda T, Schwartz JE, Shimada $\mathrm{K}$. Morning surge in blood pressure as a predictor of silent and clinical cerebrovascular disease in elderly hypertensives: a prospective study. Circulation 2003; 107: 1401-1406.

7 Kario K. Obstructive sleep apnea syndrome and hypertension: mechanism of the linkage and 24-h blood pressure control. Hypertens Res 2009; 32: 537-541.

8 Shirasaki O, Yamashita S, Kawasaki S, Tagami K, Ishikawa J, Shimada K, Kario K. A new technique for detecting sleep apnea-related 'midnight' surge of blood pressure. Hypertens Res 2006; 29: 695-702.

9 Shirasaki O, Kuwabara M, Saito M, Tagami K, Washiya S, Kario K. Development and clinical application of a new technique for detecting 'sleep blood pressure surges' in sleep apnea patients based on a variable desaturation threshold. Hypertens Res 2011; 34: 922-928.

10 The AASM Manual for the Scoring of Sleep and Associated Events. Rules, terminology and technical specifications. (Iber C, Ancoli-Israel S, Chesson AL, Quan SF). American Academy of Sleep Medicine. Westchester: IL, 2007. 